Service social

\title{
Évaluation d'un partenariat dans le cadre de la mise en place de services intersectoriels pour des enfants victimes d'agressions sexuelles
}

\section{Manon Bergeron et Marc Tourigny}

Volume 57, numéro 1, 2011

URI : https://id.erudit.org/iderudit/1006244ar

DOI : https://doi.org/10.7202/1006244ar

Aller au sommaire du numéro

Éditeur(s)

École de service social de l’Université Laval

ISSN

1708-1734 (numérique)

Découvrir la revue

Citer cet article

Bergeron, M. \& Tourigny, M. (2011). Évaluation d'un partenariat dans le cadre de la mise en place de services intersectoriels pour des enfants victimes

d'agressions sexuelles. Service social, 57(1), 1-14.

https://doi.org/10.7202/1006244ar
Résumé de l'article

Cet article vise à dégager les facteurs ayant facilité ou non le développement d'un partenariat dans le cadre de la mise en place des services intersectoriels pour les enfants victimes d'agressions sexuelles. Les résultats présentés sont issus d'entrevues individuelles et de groupe réalisées auprès du personnel des organismes partenaires impliqués dans la dispensation de services cliniques $(n=57)$. Cette étude évaluative identifie les facteurs de réussite et les embûches rencontrés qui sont principalement liés à l'environnement, à la structure, à la dynamique du groupe et à l'efficacité du partenariat. 


\title{
Évaluation d'un partenariat dans le cadre de la mise en place de services intersectoriels pour des enfants victimes d'agressions sexuelles
}

\author{
Manon Bergeron \\ Marc Tourigny
}

\section{RÉSUMÉ}

Cet article vise à dégager les facteurs ayant facilité ou non le développement d'un partenariat dans le cadre de la mise en place des services intersectoriels pour les enfants victimes d'agressions sexuelles. Les résultats présentés sont issus d'entrevues individuelles et de groupe réalisées auprès du personnel des organismes partenaires impliqués dans la dispensation de services cliniques $(n=57)$. Cette étude évaluative identifie les facteurs de réussite et les embûches rencontrés qui sont principalement liés à l'environnement, à la structure, à la dynamique du groupe et à l'efficacité du partenariat.

Mots clés : Évaluation; partenariat; organisation des services; enfants victimes d'agressions sexuelles.

\section{Problématique}

Contexte québécois concernant les services aux enfants victimes d'agressions sexuelles Au cours des vingt dernières années, le Québec a réalisé une réflexion importante sur la question des agressions sexuelles à l'égard des enfants. Dans la foulée des recommandations du Groupe de travail sur les agressions à caractère sexuel (1995), le Québec s'est doté d'orientations en matière d'agression sexuelle et d'une entente multisectorielle pour s'attaquer à ce problème touchant une proportion importante des enfants québécois (Gouvernement du Québec, 2001a; 2001b). Essentiellement, cette entente multisectorielle a pour but d'améliorer la concertation et la coordination entre les différents organismes québécois impliqués dans les situations où des enfants sont victimes d'agression sexuelle, d'abus physique ou de négligence grave. Cette entente est le résultat d'une analyse ayant mis en évidence certains constats, dont des problèmes importants de communication entre les différentes organisations, des règles de confidentialité ambiguës qui restreignent la concertation et des interventions morcelées. Depuis 2001, les organismes impliqués (centres jeunesse, services policiers, centres hospitaliers, écoles, etc.) ont entrepris la mise en œuvre de cette entente et l'appliquent partout au Québec.

L'un des principaux constats émis dans les Orientations gouvernementales en matière d'agression sexuelle était la nécessité de déployer d'autres efforts afin d'améliorer les services psychosociaux, médicaux, judiciaires et policiers auprès des enfants agressés sexuellement et de leur famille (Gouvernement du Québec, 2001b). Parmi les moyens proposés, notons: 1) l'implantation et la consolidation des programmes de traitements dont l'efficacité est reconnue ou qui apparaissent prometteurs; et 2) la mise en place ou la consolidation d'une gamme de services (accueil, information, soins médicaux, aide psychosociale individuelle et de groupe). 
C'est dans ce contexte qu'une fondation privée, de même qu'une douzaine d'organismes de la grande région métropolitaine de Montréal provenant de différents secteurs, se sont regroupés afin de développer un centre d'expertise en agression sexuelle pour les enfants âgés de 12 ans et moins et pour leur famille. Ce centre d'expertise est un organisme québécois sans but lucratif (créé au printemps 2005) dont la mission est, entre autres, de développer l'expertise de pointe en matière d'agression sexuelle en associant les services médicaux, psychosociaux, policiers et judiciaires à la connaissance et à la recherche. Les trois champs d'activité du centre d'expertise sont les services cliniques, la formation et la recherche. Ce partenariat vise l'atteinte de plusieurs objectifs dont les suivants: rassembler en un seul lieu tous les services dont les enfants victimes d'agression sexuelle et leurs proches peuvent avoir besoin suite au dévoilement d'une agression sexuelle, accroître l'accessibilité et l'efficacité des services offerts et développer des services thérapeutiques spécialisés. En visant ces objectifs, le principal avantage présumé consiste en une meilleure intégration, continuité et adaptation des services aux besoins des enfants victimes d'agression sexuelle.

L'ensemble des organismes a consenti à procéder à une évaluation de l'implantation des services cliniques dans le cadre du partenariat établi autour du centre d'expertise. Cette étude ${ }^{1}$ a été réalisée à la deuxième et la troisième année d'implantation du centre d'expertise, soit de septembre 2006 à octobre 2008, et elle visait trois objectifs : 1) décrire la participation de chacun des principaux partenaires au processus de gestion clinique du centre d'expertise; 2) décrire la participation des principaux partenaires dans la dispensation des services; 3) dégager les processus ayant facilité ou non le développement de ce partenariat dans la mise en place de l'ensemble des services intersectoriels pour les enfants victimes d'agressions sexuelles. Les résultats présentés dans cet article sont essentiellement liés à ce dernier objectif. La prochaine section vise à préciser certains aspects du partenariat dont il est question dans cette étude, informations qui permettent de saisir les particularités de cette organisation des services.

\section{Caractéristiques du partenariat mis en place au centre d'expertise}

Les organismes impliqués dans ce partenariat proviennent presque exclusivement des régions de Montréal, de Laval et de la Montérégie, avec une représentation importante pour celle de Montréal. Initialement quatre secteurs étaient représentés par les partenaires : les secteurs de la santé, judiciaire, policier et social. À ces secteurs s'ajoute celui du milieu universitaire pour le volet recherche (chaire interuniversitaire regroupant une équipe de chercheures et chercheurs dont les recherches sont liées aux agressions sexuelles). Le nombre de partenaires s'est accru entre le début du projet et la fin de l'étude et un seul organisme s'est retiré de ce partenariat.

1. Cette étude a bénéficié d'un financement du Centre national de la prévention du crime (Sécurité publique du Canada) et de la Chaire interuniversitaire Fondation Marie-Vincent sur les agressions sexuelles envers les enfants. 
Un comité permanent réunissant tous les partenaires a été mis sur pied dès le début du partenariat en avril 2005: cette Table des partenaires (après dans ce texte: TP) était initialement désignée comme le lieu regroupant des gestionnaires ${ }^{2}$ de tous les organismes partenaires à raison de quatre ou cinq réunions par année. Le mandat de ce comité stipule que cette instance consultative permet de valider les orientations du centre d'expertise puis de soutenir la concertation au niveau des activités intersectorielles et des services offerts. En plus de la participation à ce comité, les partenaires étaient également sollicités pour d'autres comités ad hoc et des réunions hors comité dans le but d'arrimer les services cliniques.

Au niveau des services cliniques, ces organismes se sont engagés dans un partenariat qui visait, notamment, à offrir aux enfants victimes d'agression sexuelle et à leurs proches la possibilité de recevoir tous les services dans les locaux du centre d'expertise. Durant la période de l'étude, les services suivants ont été implantés: l'investigation policière (entrevue avec la victime présumée), l'investigation médicale (examen médical de l'enfant comprenant son bilan de santé), l'évaluation des besoins psychosociaux de l'enfant et des parents non agresseurs (évaluation des conséquences associées à l'agression sexuelle et validation du besoin de traitement pour chaque enfant), la dispensation d'un traitement psychosocial aux enfants et à leur famille et l'accompagnement des parents dans les différentes démarches suivant l'investigation policière. Ces services sont devenus graduellement accessibles à partir d'avril 2006.

Les organismes partenaires n'ont pas tous la même implication dans la dispensation des services. En effet, certains partenaires assurent une présence permanente (temps plein ou temps partiel) au centre pour dispenser un service : le service de police pour les investigations policières, le centre hospitalier pour les investigations médicales et le centre d'expertise luimême pour l'ensemble des services psychosociaux. D'autres partenaires utilisent les locaux ponctuellement mais sans assurer de présence permanente pour des rencontres avec les enfants, comme les centres jeunesse dans le cadre de l'entente multisectorielle. Pour certains partenaires, leur implication se résume à accompagner un enfant au centre pour l'un ou l'autre des services offerts. Enfin, d'autres partenaires sont exclusivement impliqués au niveau de la référence (il est à noter que tous les partenaires peuvent faire de la référence, peu importe leur niveau d'implication).

\section{Cadre conceptuel pour l'évaluation du partenariat}

La notion de partenariat est présente depuis quelques années dans plusieurs sphères d'activité, notamment dans le milieu scolaire, les services sociaux, l'industrie et l'administration. À titre d'exemple, l'établissement d'un partenariat s'observe dans les domaines de la réadaptation en déficience intellectuelle et physique (Kalubi, Boudreault et Bouchard, 2005), de la santé mentale (Boydell, 2003) et de la délinquance juvénile (Minkes, Hammersley et Raynor, 2005). Schulz, Israel et Lantz (2003) suggèrent que le partenariat peut être défini comme un regroupement d'individus ou d'organismes (nommés partenaires) qui s'unissent dans le but de réaliser un

2. Le terme gestionnaire signifie ici que l'individu occupe une fonction qui inclut la responsabilité d'un service ou d'une équipe. 
projet ou un mandat visant à promouvoir le bien-être, à prévenir ou à réduire les conséquences d'un problème dans une communauté donnée. Selon ces mêmes auteures, le partenariat implique les deux prémisses suivantes : 1) divers partenaires contribuent par leurs différentes perspectives, expertises et ressources à l'identification et à la compréhension d'un problème social commun; 2) ces perspectives et ces ressources sont engagées dans le développement et la mise en place de solutions au problème social. Dans le même sens, Mérini (2002, p. 142) définit le partenariat comme ceci : «agir de concert et en complémentarité à partir des différences de chacun pour résoudre un problème reconnu comme commun dans un ensemble de synergies et d'intérêts organisés ».

Les avantages que peut présenter le partenariat pour les organisations peuvent être de rehausser l'efficacité, de mettre en commun les ressources et l'expertise, d'éliminer le dédoublement, de faciliter la coordination des interventions, de partager la responsabilité du résultat ainsi que de maximiser le pouvoir d'action et l'utilisation des ressources existantes (Boydell, 2003; Kegler, Steckler, McLeroy et Malek, 1998; Schulz et al., 2003). En réponse à un problème social, le partenariat constitue, selon Mérini (2002, p. 144), un moyen pour accentuer la proximité entre les organisations et les individus afin d'accélérer les prises de décision et « d'augmenter leur zone de liberté et de possibilités d'action en utilisant leurs différences comme des complémentarités ».

Bien que les avantages du partenariat soient largement diffusés, les défis ou les difficultés possibles semblent moins documentés. Or, dans sa recension des écrits scientifiques, Boydell (2003) énumère les principaux facteurs qui contribuent aux succès ou aux difficultés rencontrées dans un processus de partenariat. Ces facteurs peuvent être liés au contexte communautaire (objectif commun, structure, mode de fonctionnement, processus décisionnel...), à la dynamique du partenariat (communication, efficacité des réunions, satisfaction des partenaires, sentiment d'efficacité au regard des objectifs, coûts/bénéfices reliés à la participation au projet, degré d'engagement...), à l'atteinte d'objectifs internes (conflits possibles, resserrement des liens entre organismes...) et à l'atteinte d'objectifs externes (nombre et type d'activités mises en place...).

La présente étude a pris en considération deux modèles comme cadre de référence théorique dans les différentes étapes de la cueillette des données et de l'analyse de ces dernières. Le modèle conceptuel de Schulz et ses collègues (2003), adapté de Sofaer (2000), permet d'évaluer la dynamique de groupe comme facteur de l'efficacité du partenariat. II repose sur une recension des modèles conceptuels et des recherches empiriques. Ce modèle est illustré à la figure 1 et il regroupe cinq catégories de facteurs pouvant influencer l'efficacité d'un partenariat : les facteurs liés à l'environnement externe au partenariat, ceux liés à la structure et à la gestion, ceux liés à la dynamique du partenariat, ceux liés à l'efficacité du partenariat et les facteurs liés à l'atteinte des objectifs. Chaque flèche du modèle illustre l'influence qu'une catégorie de facteurs peut avoir sur les autres catégories pour ultimement contribuer à l'atteinte des objectifs du partenariat. Par exemple, les collaborations antérieures, établies entre les organisations avant le partenariat (facteurs liés à l'environnement), peuvent avoir un effet tant sur la structure et la gestion du partenariat que sur la dynamique de groupe et la mise en place des services. 
Par ailleurs, Kegler et ses collègues (1998) ont également élaboré un modèle conceptuel des facteurs influençant l'efficacité d'un partenariat. Selon ce modèle, les processus opérationnels du partenariat, en combinaison avec les aspects structurels et les ressources des partenaires, ont une influence sur l'efficacité du partenariat. Ce modèle précise également qu'un niveau élevé de satisfaction et de participation des partenaires entraîne une plus grande qualité des actions, une plus grande mobilisation des ressources et une meilleure implantation des services. Ces derniers éléments ont un effet direct sur l'efficacité du partenariat, c'est-à-dire l'atteinte des objectifs.

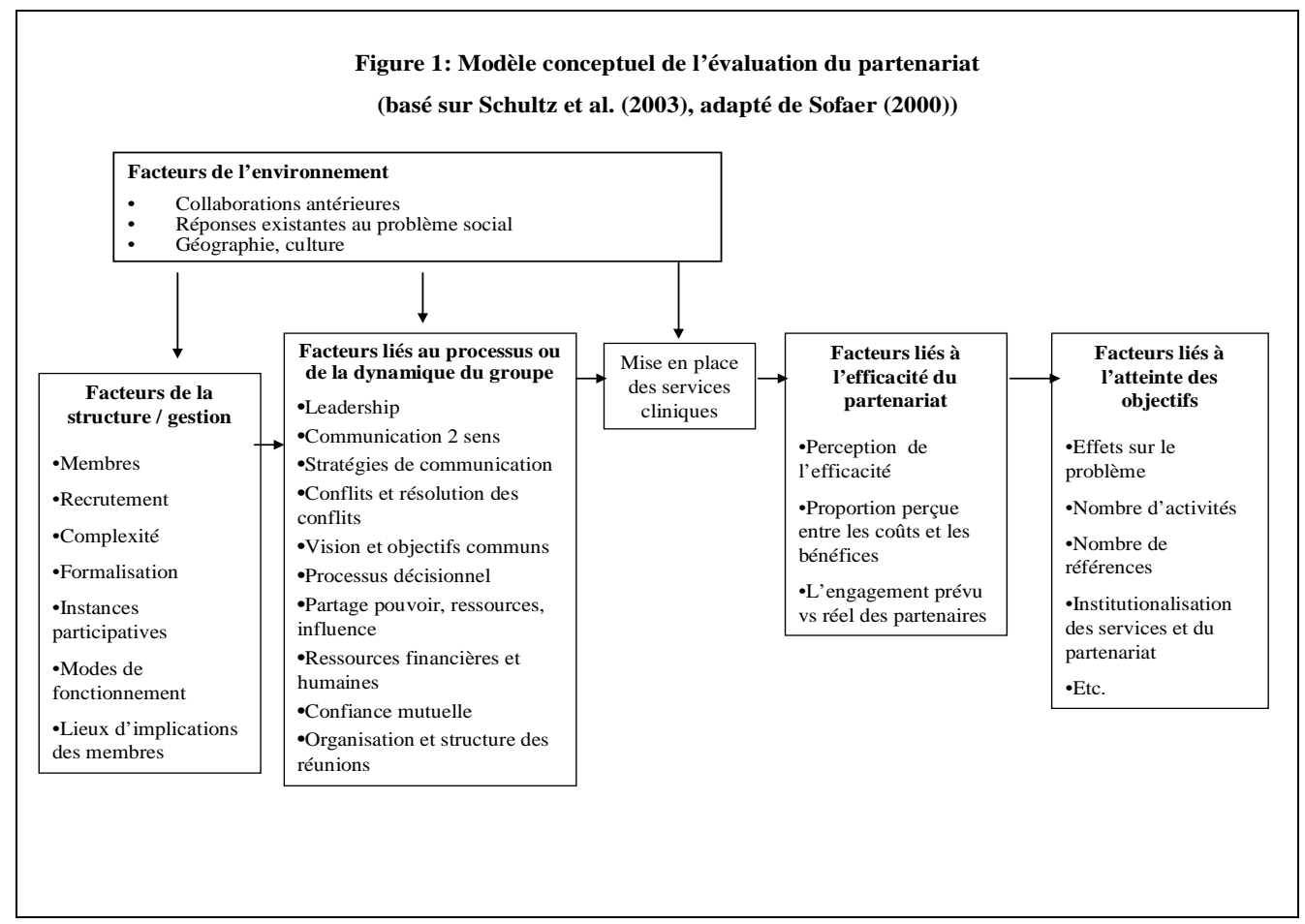

\section{MÉTHODOLOGIE}

\section{Méthodes de cueillette des données}

La méthodologie de cette étude évaluative est mixte, avec une dominance de la méthodologie qualitative. Au niveau quantitatif, la méthode retenue a été la compilation informatique des informations relatives à la dispensation des services: nombre et provenance des références, nombre d'investigations médicales, nombre d'investigations policières, nombre d'intervenantes et d'intervenants rencontrés par l'enfant, délai entre les étapes d'intervention, nombre d'enfants ayant bénéficié de plusieurs services au centre, etc. La compilation de ces informations a permis de décrire la participation réelle des partenaires dans la dispensation des services cliniques. Pour ce faire, l'équipe de recherche a conçu une banque informatisée de données dans le but de permettre de mieux décrire mieux décrire le parcours des enfants à travers les différents services offerts au centre. 
Quant au niveau qualitatif, plusieurs méthodes ont permis de recueillir les données, telles que l'analyse de documents officiels (par exemple les procès-verbaux des comités), l'observation directe des réunions de la TP (de mai 2007 à octobre 2008), 17 entrevues individuelles semi-structurées auprès des gestionnaires siégeant à ce comité (automne 2007) et 10 entrevues de groupe auprès de 40 intervenantes et intervenants (printemps 2008). La combinaison de ces quatre méthodes qualitatives a visé à dégager les facteurs ayant facilité ou freiné le partenariat.

Toutes les entrevues ont été enregistrées et retranscrites pour être analysées avec le logiciel Nvivo. Au niveau de la stratégie d'analyse des données, la position adoptée est celle de la logique inductive délibératoire (Savoie-Zajc, 2004). Cette position consiste à reconnaître l'usage et l'influence du cadre théorique dans l'analyse des données; celui-ci devient ainsi un outil qui guide le processus d'analyse tout en permettant l'émergence d'autres catégories.

\section{Le choix et le recrutement de l'échantillon}

Dans le but de discerner les facteurs ayant influencé le développement du partenariat, il convenait de documenter l'expérience des gestionnaires impliqués dans le processus de gestion des services cliniques mis en place au centre d'expertise, mais également l'expérience des intervenantes et des intervenants ayant été directement interpellés dans la dispensation des services cliniques. Étant donné que des organismes se sont joints à ce partenariat au cours de l'étude, un critère d'inclusion était nécessaire, soit celui d'être un organisme participant à la TP à l'automne 2007. La lecture du tableau 1 permet d'avoir un aperçu de la répartition des 57 répondants et répondantes, selon leur provenance et leur fonction (gestionnaires ou intervenants-es).

Tableau 1 : Liste des partenaires et répartition des répondantes et répondants

\begin{tabular}{l|c|c}
\hline Partenaires impliqués dans l'étude & Gestionnaires & Intervenants-es \\
\hline Centre d'expertise & 2 & 6 \\
\hline Centres d'aide aux victimes d'actes criminels & 3 & 10 \\
\hline Centre hospitalier & 5 & 2 \\
\hline Centres jeunesse & 2 & 14 \\
\hline Milieu policier & 1 & 6 \\
\hline Indemnités et services aux victimes d'actes criminels & 2 & 2 \\
\hline Chaire de recherche & 1 & - \\
\hline Centre de santé et des services sociaux & 17 & - \\
\hline Total de répondantes et répondants & 2,6 ans & 3,2 ans \\
\hline Délai entre le début du partenariat et les entrevues & 2,3 ans & 2,7 ans \\
\hline Ancienneté moyenne des organismes lors de l'entrevue & 1,5 an & - \\
\hline Ancienneté moyenne des gestionnaires lors de l'entrevue & & 40 \\
\hline
\end{tabular}


Ainsi, le premier groupe se compose de 17 gestionnaires délégués à la TP et impliqués dans la mise en place des services cliniques offerts aux enfants. II est à noter que tous les individus ont été sollicités, même dans les cas où un organisme partenaire avait plusieurs délégués à la TP. Pour ce premier groupe, la participation à l'étude a consisté à consentir à la présence de l'agente de recherche comme observatrice lors des réunions de la TP et à participer à une entrevue individuelle à l'automne 2007. Les aspects abordés lors de cette entrevue étaient l'implication de l'organisme du point de vue de la gestion et de la dispensation des services cliniques, les facteurs ayant facilité ou freiné le partenariat et le niveau de satisfaction en lien avec l'organisation et la structure des comités.

Le second groupe se compose de 40 intervenantes et intervenants travaillant au sein des organismes partenaires et ayant été directement interpellés dans la dispensation des services cliniques. Ces individus avaient donc expérimenté la structure des services mise en place dans ce partenariat, que ce soit par la référence, l'accompagnement, la réalisation d'investigations policières, la réalisation d'investigations médicales, l'intervention psychosociale au centre d'expertise, etc. Une entrevue de groupe a été réalisée auprès de chaque organisme impliqué dans les services cliniques au printemps 2008. Pour la majorité des organismes partenaires, la totalité des intervenantes et des intervenants qui en relevaient et collaboraient au partenariat ont été sollicités car leur nombre était inférieur à 10 personnes. Dans les organismes où plus de 10 individus avaient collaboré avec le centre d'expertise, la sélection a été effectuée de façon à maximiser la représentativité des intervenantes et des intervenants en fonction de leur rôle dans les services.

Les répondantes et répondants ont signé un formulaire de consentement. Par ailleurs, cette étude a été approuvée par le comité d'éthique de la recherche Éducation et sciences sociales de l'Université de Sherbrooke.

\section{RÉSUltats}

La présentation des résultats s'articule autour des catégories incluses dans le cadre conceptuel retenu pour cette étude. Ainsi, pour chacune des catégories, sont exposés les principaux résultats liés aux facteurs positifs ou négatifs ayant influencé le processus de partenariat dans la mise en place des services cliniques au centre d'expertise. Quelques résultats de la banque informatisée de données sont également insérés à titre d'indicateurs quantitatifs (section 3.4).

\section{Facteurs liés à l'environnement externe au partenariat}

Certains facteurs environnementaux ont contribué positivement au développement du partenariat pour l'implantation d'un centre d'expertise en agression sexuelle pour les enfants. Un facteur qui ressort particulièrement concerne les collaborations antérieures des organismes impliqués dans le partenariat actuel. En effet, avant le projet du centre d'expertise, plusieurs collaborations existaient déjà entre les organismes. L'exemple le plus évident est l'entente multisectorielle qui agissait comme une structure de coordination entre les centres jeunesse, les services de police et les procureurs, de même qu'avec d'autres organismes qui pouvaient s'y greffer selon la situation signalée. Un autre exemple est celui des partenariats de recherche ayant existé par le passé, puisque la majorité des partenaires du centre d'expertise 
ont collaboré à une ou plusieurs recherches dans le passé avec les chercheures et chercheurs de la chaire de recherche. Ainsi, plusieurs partenaires (gestionnaires, intervenantes et intervenants) ont souligné que les collaborations ou les liens antérieurs entre les organisations ou les équipes de recherche avaient assuré une continuité et un certain niveau de confort qui favorisaient l'établissement actuel du partenariat pour la mise en place des services cliniques.

Par ailleurs, le manque de temps et de ressources des organisations est un facteur qui désavantage le partenariat. Ces manques peuvent être causés par des réorganisations chez les partenaires, une surcharge de travail, du roulement de personnel, des changements de poste, etc. Les conséquences associées à ce facteur sont principalement des retards ou des ralentissements dans l'implication réelle d'un partenaire.

\section{Facteurs liés à la structure de gestion}

Au niveau de la structure de gestion, les facteurs suivants ont particulièrement facilité le processus partenarial et l'implantation des services cliniques : la composition et la stabilité du membership de même que le statut des gestionnaires et leur expertise. D'abord, les partenaires présents au début du projet détenaient tous une expertise en matière d'agression sexuelle envers les enfants, dans les différents secteurs et en recherche; aussi, les gestionnaires délégués à la TP étaient des personnes-clés connaissant les différents besoins à combler pour ce qui était des services cliniques à mettre en place. De plus, le fait que tous les secteurs étaient mobilisés dans ce partenariat assurait une complémentarité. Ensuite, à l'exception d'un organisme, les partenaires présents au début du partenariat en 2005 étaient toujours impliqués au moment de l'étude, ce qui est un indice fiable de la stabilité dans le membership. Par ailleurs, la composition initiale de la TP indique que les gestionnaires se situaient à un niveau décisionnel élevé dans leur organisation : leur présence a certainement facilité les prises de décision nécessaires à l'avancement des travaux et ainsi contribué à l'efficacité avec laquelle les services cliniques ont pu être déployés au centre d'expertise.

En revanche, au cours de l'étude, il a été possible d'observer plusieurs changements de gestionnaires délégués à la TP, soit parce qu'un nouveau représentant avait été nommé pour représenter l'organisme à la TP, ou parce qu'un collègue avait été délégué pour une réunion afin de remplacer momentanément le représentant régulier. Cette instabilité des gestionnaires à la TP est un facteur négatif soulevé par des répondantes et répondants. Les impacts notés de ces changements sont principalement une obligation de faire de fréquentes mises à jour des informations, une réduction de la qualité des suivis et une moins bonne transmission des informations entre les organisations.

\section{Facteurs liés au processus ou à la dynamique du partenariat}

Les résultats sont nombreux en ce qui concerne les facteurs liés au processus ou à la dynamique de groupe. Parmi les indicateurs de réussite liés à la dynamique du groupe se trouve le partage d'une vision et d'objectifs communs. En effet, le partenariat est favorisé lorsque les membres voient les choses de façon semblable et s'accordent sur les mêmes objectifs pour tendre vers un but commun. Le constat général qui se dégage des deux groupes de répondantes et répondants est sans conteste que les organismes sont mobilisés par un 
projet qu'ils perçoivent comme rassembleur et porteur d'espoir. L'objectif « d'accroître l'accessibilité, la qualité et l'efficacité des services offerts » pour les enfants victimes d'agression sexuelle est un élément rassembleur tout particulièrement pour les gestionnaires. Par ailleurs, l'objectif de « rassembler en un seul endroit tous les services dont les victimes d'agression sexuelle et leur famille peuvent avoir besoin » est un élément rassembleur chez les intervenantes et les intervenants parce qu'il est perçu comme l'espoir d'une continuité de services et d'une accessibilité plus rapide aux services.

D'un autre côté, un facteur qui ressort de façon marquée concerne la communication. Sur ce point, le niveau de satisfaction des gestionnaires diffère de celui des intervenantes et des intervenants. D'abord, les premiers ont exprimé leur satisfaction face aux informations qui circulent et ils s'estiment généralement bien renseignés et tenus informés des changements. Cependant, l'expérience des intervenantes et des intervenants interrogés démontre certaines difficultés dans la diffusion de l'information. En effet, malgré le fait que ces individus aient tous été directement en contact avec le centre d'expertise, plusieurs ont noté un manque de connaissances quant au rôle des partenaires impliqués dans le projet (exemple : qui fait quoi, quels sont les liens entre les organismes). De plus, plusieurs de ces intervenantes et intervenants constataient une insuffisance d'informations quant au fonctionnement concret des services offerts aux enfants (par exemple : les mécanismes de référence au centre d'expertise, la trajectoire typique d'un enfant à travers les services).

Ainsi, durant la période de l'étude, les mécanismes de communication utilisés ne semblaient pas suffisants, autant au niveau du fonctionnement général du centre d'expertise qu'au niveau des échanges d'informations cliniques entre les partenaires. Selon les participants, cette insuffisance des informations peut nuire directement au bon fonctionnement de l'implantation des services, notamment en termes de non-référence ou de mauvaises procédures de référence. Les intervenantes et les intervenants des partenaires soulignent presque unanimement la nécessité d'assurer une diffusion de l'information qui soit efficace et régulière. Sur le plan clinique, les échanges d'informations pour un enfant spécifique semblent parfois poser des difficultés pour diverses raisons: un manque de rapidité dans les réponses entre partenaires, un manque d'information clinique concernant l'évolution des enfants orientés au centre d'expertise.

Enfin, un résultat qui touche davantage la nature de ce partenariat (présence d'organisations de différents secteurs) concerne l'importance pour les partenaires d'adapter leurs pratiques en raison de l'influence ou de la contribution de différentes expertises. Lors des entrevues individuelles, des gestionnaires ont noté que des efforts doivent être faits afin d'éviter que chaque secteur ne reste campé dans ses habitudes et ses procédures. Bien plus, pour certains gestionnaires, les secteurs doivent apprendre à partager un espace commun dans lequel chaque secteur " empiète " un peu sur le champ d'expertise des autres et accepte que les autres détiennent aussi une expertise en vertu de laquelle ils empiètent sur son propre champ d'expertise. À titre d'exemple, cela peut signifier que les enquêteurs du service de police remettent en question la nécessité des informations recueillies par l'équipe médicale, ou à l'inverse, que l'équipe médicale interroge le service de police sur son hésitation à lui transmettre certaines informations qu'elle juge pertinentes pour le secteur médical. Il apparaît donc que le 
travail intersectoriel peut entraîner des modifications dans les pratiques respectives. C'est ainsi que dans la dynamique de ce partenariat, la présence des différents secteurs de pratique est perçue comme un facteur de réussite mais elle demeure également un défi important pour le futur.

\section{Facteurs liés à l'efficacité du partenariat et à l'atteinte des objectifs}

L'un des principaux constats de l'étude est le niveau élevé de participation et de mobilisation des partenaires pour la mise en place des services au centre d'expertise. Les résultats montrent en effet que les partenaires se sont engagés et mobilisés de façon importante et constante depuis le début du partenariat en 2005 et jusqu'à la fin de l'étude. Par exemple, le taux élevé de participation des organismes à la TP (taux moyen annuel de présence des organismes à la TP : $83 \%$ pour 2006-2007 et $87 \%$ pour 2007-2008) et le faible taux d'abandon des partenaires au cours de l'étude (un seul partenaire a cessé de collaborer) sont des indicateurs de cet engagement réel.

Par conséquent, cette mobilisation a eu un effet direct sur l'implantation des services cliniques au centre d'expertise. En effet, les résultats concernant les services offerts au cours des deux années de l'étude montrent qu'il y a eu une implantation de la plupart des services prévus et que la mise en place de ces services nécessitait une implication concrète de plusieurs partenaires. Ces services sont l'investigation policière, l'investigation médicale et les services psychosociaux (principalement l'évaluation psychosociale et le traitement des enfants agressés sexuellement ou des enfants présentant des comportements sexuels problématiques).

De plus, cette mobilisation des partenaires s'est maintenue et, pour certains, elle s'est accentuée. Notamment, les résultats montrent une hausse importante, entre l'année 2006-2007 et celle de 2007-2008, du nombre d'investigations policières réalisées (85 à 132), d'investigations médicales réalisées (11 à 33) et des demandes reçues pour les services psychosociaux grâce à la référence des partenaires (86 à 170). Un autre exemple est celui de l'augmentation du nombre d'heures hebdomadaires de présence au centre d'expertise de certains partenaires afin d'offrir des services à cet endroit, notamment le centre hospitalier et le service de police.

La satisfaction des gestionnaires était très élevée à l'égard de l'état des travaux, particulièrement pour un projet aussi complexe dans lequel il est normal que la présence de différents secteurs ralentisse le processus. De même, les intervenantes et les intervenants interrogés perçoivent les services au centre d'expertise comme bénéfiques pour les enfants qui sont susceptibles de recevoir une gamme diversifiée de services. Il est donc possible de conclure que les intervenantes et intervenants sont également satisfaits de la mise en place des services cliniques, malgré les coûts engendrés par ce partenariat pour leur organisation et pour eux-mêmes (par exemple, les nouvelles pratiques exigent plus de temps que les anciennes procédures, l'implication d'un plus grand nombre d'intervenantes et d'intervenants auprès d'un enfant). 
Malgré cette satisfaction, la cohésion entre les partenaires n'est pas toujours ressentie par le groupe des intervenantes et des intervenants. En effet, certaines et certains ont l'impression que la collaboration actuelle entre les partenaires ne représente pas encore un réel partenariat, en ce sens qu'il persiste un cloisonnement entre les équipes des différents secteurs. Ce cloisonnement semble attribuable au déficit d'échanges d'informations cliniques entre les secteurs, au temps retreint pour les réunions intersectorielles et aux relations interpersonnelles encore à bâtir. Bref, la cohésion entre les différents secteurs de pratique est en construction et elle constitue un défi non négligeable.

\section{DISCUSSION ET RECOMMANDATIONS}

II ne fait pas de doute que la participation active et la mobilisation maintenue des partenaires sont liées au fait que le projet d'un centre d'expertise en matière d'agression sexuelle pour les enfants a constitué un projet rassembleur et porteur d'espoir. Tel que le rappelle le modèle théorique, l'un des ingrédients essentiels d'un partenariat efficace est la présence d'un but commun. II est toutefois intéressant de noter que ce but commun peut être perçu sous différents angles chez les partenaires: il peut signifier accroître l'accessibilité à une ressource, offrir différents services sous un même toit ou améliorer la qualité des services. Par ailleurs, bien que la notion d'«amélioration de la qualité des services» soit largement endossée par les partenaires, il n'a pas été possible au cours de l'étude de repérer les critères qui puissent la définir.

Tel que mentionné précédemment, Schulz et al. (2003) soulignent que le partenariat implique l'apport par les partenaires de leurs différentes perspectives, de leurs expertises et de leurs ressources pour l'identification des besoins et la compréhension des besoins identifiés, que ces multiples perspectives et ressources peuvent efficacement permettre de développer des solutions mieux adaptées aux besoins. Cette conclusion de Schulz et al. (2003) implique donc des mises en commun et un partage de l'expertise. En d'autres termes, la mise en place du partenariat au centre d'expertise se veut un projet permettant d'offrir des services médicaux, judiciaires et psychosociaux intégrés les uns aux autres. Il ne s'agit donc pas simplement de faire travailler différents secteurs dans un même lieu sans modifier les façons de faire de ces différents secteurs. Ainsi, les partenaires doivent investir un espace commun dans lequel les pratiques respectives sont sujettes à être modifiées en raison de la présence des différentes expertises. Ces réflexions rejoignent deux principes émis par Mérini (2002), soit le principe de la rupture et celui du risque. Le principe de la rupture suppose que le partenariat entraîne une coupure avec les schèmes habituels d'organisation des services. Quant au principe du risque, il fait référence aux risques attribuables à « l'ouverture et à la présence du regard de l'autre et à des logiques différentes » (p. 147).

Or, il semble que le partage d'expertise dans ce partenariat demeure actuellement un idéal à atteindre plutôt qu'une réalité terrain. L'un des indicateurs potentiels qui pourrait traduire ce constat est la proportion d'enfants bénéficiant de plusieurs services au centre d'expertise. Plus précisément, les résultats montrent que malgré le fait que plusieurs services des différents partenaires soient implantés au centre d'expertise, peu d'enfants (16\%) bénéficiaient au 
moment de l'étude de plusieurs services au centre d'expertise impliquant plusieurs partenaires (Bergeron et Tourigny, 2009). Ainsi, le partage de l'expertise à travers l'ensemble des dossiers n'était pas très présent puisque dans la majorité des dossiers au centre d'expertise, un seul partenaire était impliqué auprès de l'enfant. Le défi pour les prochaines années semble donc être d'accroître ce partage de l'expertise entre les différents partenaires. Ceci implique de réfléchir à la notion même d'expertise et aux moyens pour développer une expertise commune.

Enfin, les résultats soulignent qu'un des aspects pouvant faire l'objet d'une amélioration concerne les stratégies de communication. À cet effet, la TP pourrait dans un premier temps échanger sur les stratégies de communication actuellement en place dans chacune des organisations. II ressort que le niveau d'information souhaité est différent d'un partenaire à l'autre, c'est-à-dire qu'un partenaire dont l'implication consiste à référer au centre d'expertise n'a pas les mêmes attentes qu'un partenaire qui collabore de manière plus étroite à la dispensation des services au centre d'expertise. C'est pourquoi, dans un deuxième temps, il pourrait être utile de se doter d'une stratégie générale de communication qui identifierait quelles sont les informations à diffuser, à qui, à quelle fréquence et comment le faire.

Également, il semble important de définir des protocoles clairs, dont on s'assurera qu'ils soient connus par l'ensemble des intervenantes et des intervenants susceptibles d'être impliqués dans la dispensation des services cliniques. Ce constat rejoint l'observation de Minkes et ses collaborateurs (2005) dans leur recension de 25 projets de partenariat sur la qualité des partenariats établis autour des services pour les délinquants juvéniles ayant des problèmes de toxicomanie. En effet, ces auteurs concluent que l'un des principaux facteurs de réussite des partenariats est la négociation rapide des ententes à propos des références, de la confidentialité et du partage des informations, de l'harmonisation des pratiques des organisations de même que des mécanismes de réponse rapide et de retour d'information aux intervenants ayant référé un enfant.

\section{FORCES ET LIMITES DE L'ÉTUdE}

Dans un premier temps, l'étude présente l'avantage d'avoir utilisé des méthodes variées de collecte de données. Également, une autre force de l'étude a consisté à recueillir l'information auprès de diverses sources, soit l'ensemble des gestionnaires impliqués à la TP et un échantillon important des intervenantes et des intervenants de ces partenaires impliqués dans la dispensation de services cliniques. Dans ce sens, les propos recueillis sont représentatifs de ces partenaires au moment de l'étude. Par ailleurs, la présente étude comprend une période de cueillette des données de deux ans, soit de septembre 2006 à octobre 2008. Cet échéancier était assurément un avantage car il offrait l'opportunité de décrire le processus partenarial durant la deuxième et la troisième année d'implantation des services au centre d'expertise.

Néanmoins, l'étude présente également des limites méthodologiques. Une limite importante à considérer lors de l'examen des résultats est le fait que ces résultats reflètent la perspective des partenaires durant les deux années de cueillette des données de l'étude. Comme nous l'avons précisé précédemment, le partenariat et la dispensation de services sont en évolution 
depuis le début du centre d'expertise. En bref, les résultats reflètent l'état de la situation au moment de l'étude. Cette limite est par ailleurs partagée par l'ensemble des études évaluatives portant sur un processus partenarial.

\section{Conclusion}

La présente étude s'est intéressée tout particulièrement à l'évaluation du processus de partenariat dans le cadre de l'implantation d'un centre d'expertise en agression sexuelle pour les enfants âgés de 12 ans et moins, plus spécifiquement durant la deuxième année et la troisième année d'implantation de ce centre. Notamment, l'étude a permis d'identifier des facteurs de réussite mais aussi des embûches rencontrées, tant du point de vue des gestionnaires que de celui des intervenantes et des intervenants. Sur la base du modèle théorique de Schulz et al. (2003) et de celui de Kegler et al. (1998), l'étude a permis de colliger des informations liées aux différentes catégories de facteurs associés à l'évaluation d'un processus partenarial.

En conclusion, l'étude fait état d'un partenariat dynamique et mobilisé dans l'implantation d'une gamme de services visant à réduire les conséquences des agressions sexuelles vécues par les enfants. Le but ultime est donc d'en arriver à mieux répondre aux besoins de ces enfants et de leur famille. Dans ce sens, le partenariat devra dans les prochaines années orienter ses efforts non seulement vers la mise en place des services, mais également vers une évaluation de leur efficacité et de la «plus-value » de ces services par rapport aux services existants avant le partenariat. II faudra donc réfléchir davantage au bien-fondé de ces services et à ce qu'ils peuvent apporter dans l'amélioration de la réponse aux besoins de ces familles.

Manon Bergeron Professeure, Département de sexologie Université du Québec à Montréal Maîtrise en sexologie complétée Doctorante en sciences de l'éducation

Marc Tourigny

Professeur, Département de psychoéducation Université de Sherbrooke Doctorat en psychologie communautaire 


\section{RÉFÉRENCES}

Bergeron, M. et M. Tourigny (2009). Évaluation de partenariat existant dans le cadre de la mise en place des services au Centre d'expertise Marie-Vincent, Rapport de recherche remis au fonds de Stratégie nationale pour la prévention du crime, Longueuil, Université de Sherbrooke.

Boydell, K. M. (2003). Overview of the current literature on coalition development, Toronto (ON), Coalition canadienne pour la santé mentale des personnes âgées.

Gouvernement du Québec (2001a). Entente multisectorielle relative aux enfants victimes d'abus sexuels, de mauvais traitements physiques ou d'une absence de soins menaçant leur santé physique, Québec, Gouvernement du Québec.

Gouvernement du Québec (2001b). Orientations gouvernementales en matière d'agression sexuelle, Québec, Gouvernement du Québec.

Groupe de travail sur les agressions à caractère sexuel (1995). Les agressions sexuelles: STOP, Québec, Gouvernement du Québec.

Kalubi, J. C., P. Boudreault et J.-M. Bouchard (2005). Partenariat entre intervenants et parents: expérience du Centre montérégien de réadaptation, Rapport de recherche du Groupe inter-réseaux de recherche sur l'adaptation de la famille et de son environnement (GIRAFE), St-Hubert.

Mérini, C. (2002). « Le partenariat : de la notion au processus », dans V. Guerdan, J. M. Bouchard et M. Mercier (dir.), Partenariat, chercheurs, praticiens, familles - De la recherche d'un partenariat à un partenariat de recherche, Montréal, Éditions Logiques, p. 141-152.

Minkes, J., R. Hammersley et P. Raynor (2005). «Partnership in working with young offenders with substance misuse problems », The Howard Journal, 44(3), p. 254-268.

Savoie-Zajc, L. (2004). « La recherche qualitative/interprétative en éducation », dans T. Karsenti et L. Savoie-Zajc (dir.), La recherche en éducation : étapes et approches, Sherbrooke, Éditions du CRP, p. 123-150.

Schulz, A. J., B. A. Israel et P. Lantz (2003). « Instrument for evaluating dimensions of group dynamics within community-based participatory research partnerships », Evaluation and Program Planning, 26(3), p. 249-262.

Sofaer, S. (2000). Working together, moving ahead: A manual to support effective community health coalitions, New York, School of Public Affairs, Baruch College, University of New York. 